\title{
Evolução e tendências das pesquisas sobre t-learning: um mapeamento das publicações disponíveis na base de dados SCOPUS de 2000 a 2011
}

Claudia Alexandra de Souza Pinto, UFSC - claudiapinto@egc.ufsc.br Airton Zancanaro, Universidade Federal de Santa Catarina - airtonza@ gmail.com Paulo Sá, Sistema Catarinense de Comunicação - paulosa@ iscc.com.br

Fernando José Spanhol, UFSC - spanhol@led.ufsc.br Fernando Antonio Crocomo, UFSC - fernanocrocomo@gmail.com

\section{Resumo:}

Estudos sobre aplicações e serviços para TV Digital Interativa com foco na educação a distância, denominados de t-learning são relativamente recentes, pois o corpo de conhecimentos na área é encontrado de forma dispersa e fragmentada. Obter uma base sólida de conhecimentos sobre t-learning apresenta-se como um desafio e uma oportunidade de pesquisa. O objetivo deste artigo é mapear o perfil das pesquisas acadêmico-científicas em t-learning. Para tanto, foram utilizados procedimentos e técnicas bibliométricas, a partir da base de dados SCOPUS, de forma a localizar estudos sobre o tema no período de 2000 a 2011. Os resultados apresentam uma caracterização das instituições, dos pesquisadores e das áreas do conhecimento que estão estudando o tema, sendo significativa a presença de pesquisadores das áreas tecnológicas, assim como uma análise geral do campo de estudos com base nos 65 artigos mapeados na pesquisa.

Palavras-chave: educação a distância, TV digital, $t$-learning, bibliometria.

\section{Evolution and trends of research on t-learning: a mapping of available publications in the SCOPUS database from 2000 to 2011}

\begin{abstract}
:
The studies about applications and services for Interactive Digital TV that focus on distance education, commonly named t-learning, are relatively recent as a consequence of the body of knowledge in the field is dispersed and fragmented. To obtain a solid theoretical foundation of the knowledge about t-learning it is presented as a challenge and an opportunity for research. The aim of this paper is to map the profile of the academic scientific research in t-learning. For this, by means of bibliometrics techniques and procedures, there were searched studies on t-learning in the SCOPUS database, within the period of 2000 to 2011. The results show a precise characterization of institutions, areas of knowledge and researchers who are studying t-learning, with significant presence of researchers in the areas of technology, as well an general overview of the field of study, based on 65 paper abstracts mapped in the survey.
\end{abstract}

Keywords: distance education, digital TV, t-learning, bibliometrics.

\section{Introdução}

O desenvolvimento acelerado das tecnologias digitais e a intensificação dos fluxos midiáticos transnacionais configuram as transformações mais significativas para o processo histórico da televisão e dos meios de comunicação como um todo (FECHINE; FIGUERÔA, 2010). Em vista disto, a televisão se inclui neste processo com a 
digitalização de sua cadeia de valor, cuja transição da TV analógica para a TV digital tem impactos desde os estúdios até os telespectadores.

As primeiras pesquisas que vieram a culminar na criação de um sistema de televisão digital datam da década de 80 e iniciaram nos Estados Unidos, com a criação do padrão de transmissão ATSC que passou a operar em outubro de 1998 (BOLAÑO e VIEIRA, 2004).

No Brasil, as transmissões digitais iniciaram em 2007, como desdobramento das decisões políticas e econômicas tomadas para a escolha do padrão tecnológico a ser utilizado no país, que instituiu o Sistema Brasileiro de Televisão Digital Terrestre (SBTVD-T), cujos objetivos visam proporcionar a interatividade e consequentemente o desenvolvimento de novas aplicações e serviços. Por meio do acesso a serviços, via controle remoto da TV, espera-se que possa haver comunicação direta com órgãos de governo (t-government) e a formação de uma rede de educação à distância (t-learning) entre outros serviços (BARBOSA FILHO, 2007; CROCOMO, 2007; ZUFFO, 2006).

Tantos os estudos sobre TV Digital interativa quanto sobre aplicações ou serviços em t-learning são assuntos relativamente novos, cujo corpo de conhecimentos ainda se encontra de forma dispersa e fragmentada. Como a televisão é um meio de comunicação, pode ser estudada pelas áreas de teorias da comunicação e das mídias; em termos de aspectos técnicos ficam a cargo das engenharias e das ciências da computação. Nesse sentido, obter uma base sólida de conhecimentos sobre t-learning de forma a mapear o campo das pesquisas neste tema apresenta-se como um desafio e uma oportunidade de pesquisa.

Assim, o presente artigo objetiva mapear o perfil das pesquisas acadêmicocientíficas em t-learning. Para tanto, foram utilizados procedimentos e técnicas bibliométricas, a partir da base de dados SCOPUS, de forma a localizar estudos sobre o tema no período de 2000 a 2011.

$\mathrm{Na}$ seção 2 são apresentadas algumas premissas teóricas sobre TV Digital e $t$ learning; a seção 3 apresenta o objetivo e escopo da pesquisa nos procedimentos metodológicos; na seção 4 está a apresentação e discussão dos resultados da pesquisa, bem como, as contribuições e sugestões para aprofundamentos dos estudos sobre $t$ learning, seguidos da conclusão.

\section{TV Digital e t-learning: aspectos conceituais}

Inicialmente, ressaltamos que a TV Digital apresenta-se como uma área de pesquisa de interesse crescente, principalmente nos países que ainda não definiram ou estão em fase de definição de seus padrões de transmissão. Alguns detalhes sobre a TV Digital são necessários para compreender melhor as motivações desta pesquisa.

Dentre as inovações tecnológicas da TV Digital que foram determinantes para a sua implementação, estão a TV de alta definição (High Definition Television). Essa tecnologia, conhecida pela sigla HDTV, começou no Japão nos anos 1980 com o propósito de oferecer melhor qualidade de imagem e som semelhante ao do cinema, fomentando estudos na Europa e nos Estados Unidos (BOLAÑO e VIEIRA, 2004).

A concepção de um sistema de TV totalmente digital, batizado de DTV (Digital Television) iniciou-se nos Estados Unidos em 1987. Com a DTV é possível a transmissão, a recepção e a exibição de conteúdo totalmente digital, que pode ser em HDTV ou não, bem como possibilita interatividade do telespectador com a programação, conhecida como Interactive Digital Television (iDTV, TVDI ou TVDi). No entanto, a possibilidade de interação depende do padrão de transmissão adotado, 
pois cada padrão tem peculiaridades quanto às opções de tecnologia de transmissão, de compressão de dados e de disponibilidade de serviços interativos.

Atualmente existem três padrões em operação no mundo: o ATSC, norteamericano em operação desde outubro de 1988; o DVB-T, europeu que começou a funcionar no Reino Unido em 1999 e o ISDB-T desenvolvido pelo Japão e implementado em 2003 (CROCOMO, 2007; BOLAÑO e VIEIRA, 2004).

O Brasil começou suas transmissões digitais em 2007 em São Paulo, optando por desenvolver um sistema híbrido, baseado no padrão japonês e aqui denominado ISDBTB. De acordo com Barbosa Filho (2007), as decisões políticas e econômicas tomadas para a escolha do padrão tecnológico a ser utilizado no país que culminaram no Decreto no 5820 de 26/7/2006 basearam-se em diversas análises do Comitê de Desenvolvimento do Sistema Brasileiro de Televisão Digital Terrestre (SBTVD-T).

A opção pela adoção do padrão japonês foi justificada, estrategicamente, pela possibilidade de compartilhar com o Japão o sistema mais avançado de TV Digital aberta disponível, visto ter sido uma melhoria dos padrões ATSC e DVB-T. Outro benefício do padrão japonês é a mobilidade, que permite a recepção móvel em celulares e TVs portáteis sem perda da qualidade das imagens ou interferências, mesmo quando em movimento, como em trens e veículos. O padrão brasileiro tem como propósito proporcionar a interatividade e consequentemente o desenvolvimento de novas aplicações e serviços como t-government e t-learning.

A TVDI é considerada como a convergência da televisão e das TICs, abrangendo três importantes características típicas das TICs: interatividade, personalização e digitalização (LYTRAS, 2002). Enquanto Aarreniemi-Jokipelto (2005) utiliza o termo $t$ learning como definição de uma TV baseada em aprendizagem interativa, Bates (2003), diz que neste novo ambiente denominado t-learning ocorre o acesso a materiais de aprendizagem ricos em vídeo, através de um dispositivo mais parecido com a TV do que um computador.

A ideia de uma aprendizagem a distância via televisão surgiu como uma opção complementar ao ensino baseado em Tecnologias de Informação Comunicação (TICs), conhecido por e-learning, e aos tradicionais programas educacionais exibidos pela TV analógica (BELLOTTI et al, 2008).

Para Roesler et al (2010) um dos recursos fundamentais procurado pelos educadores com a utilização da TVDI é a possibilidade do estudante interagir com a programação da TV e que este deve ser um recurso didático-pedagógico a ser explorado. Esses autores desenvolveram um modelo de entrega de programas educativos via TVDI no qual os cursos são disponibilizados de forma simultânea e independente para os estudantes, sendo que os cursos podem ser armazenados no receptor de TV digital (mais comumente conhecido como set-top-box), de forma que os estudantes possam cursá-los assincronamente. Os cursos também são integrados ao ambiente de aprendizagem, no qual o estudante pode realizar atividades de aprendizagem sempre que desejar.

No curso "Vida Saudável" de Roesler et al (2010, p. 10) o estudante pode interagir com o conteúdo do curso de "forma local (interação através de animações e questionários), ou mesmo de forma global, participando de fóruns de discussão e debates descentralizados com tutores, à semelhança de cursos a distância na web".

No projeto "Trânsito Legal" desenvolvido por Dias, Rodrigues e Farias Junior (2010) os autores procuraram viabilizar uma alternativa de educação a distância por meio do uso de uma aplicação com interatividade local pela TVD. Foi desenvolvida uma aplicação sobre educação no trânsito baseada no Código de Trânsito Brasileiro, de forma a compor um rico material de apoio para os motoristas que precisam reciclar os 
conhecimentos sobre o código ou para quem estiver fazendo os exames para obtenção da primeira habilitação.

A aplicação foi estruturada sob três pontos de interação: (1) leitura da teoria sobre o trânsito; (2) testes com questões objetivas sobre a teoria disponibilizada e (3) uma área destinada ao entretenimento contendo uma lista de jogos sobre a temática da aplicação. Para testar o aplicativo, foi utilizado "um simulador de um ambiente de TV digital em computador, onde a interação homem-máquina acontece totalmente através do uso do controle remoto" (DIAS, RODRIGUES e FARIAS JUNIOR, 2010, p. 8). Foram aplicados questionários e entrevistas com 14 participantes para avaliação do aplicativo em termos dos recursos disponíveis (cursos, testes e material teórico) que tiveram uma aceitação positiva.

Gomes e Lima (2005) propõem a confecção de um material didático impresso em papel que contem links para outros materiais de estudo disponibilizados eletronicamente (vídeo, animação, imagens, exercícios etc.) que podem ser exibidos na TV. Os links são impressos na forma de código de barras no material didático em formato papel. $\mathrm{O}$ aluno poderá assistir a um vídeo que esteja disponível sobre o assunto que estiver lendo, por exemplo, caso o link para o vídeo tenha o código de barras no material impresso. A ideia central dos autores é de enriquecer a experiência do estudante durante o seu processo de aprendizagem com a utilização de materiais didáticos complementares e interligados, para motivá-lo durante os estudos. Assim, "o estudante terá o material impresso como principal fonte de conhecimento, e o material eletrônico armazenado no set-top-box servirá como um material complementar" (GOMES e LIMA, 2005, p. 7).

Gomes, Lima e Nevado (2009) desenvolveram um objeto de aprendizagem (OA) com base nas pesquisas sobre o "Gangorra Interativa" de Melo et al (2008) e realizaram experimentos com grupos de estudantes para avaliar a eficácia da utilização do OA para aprendizagem. Neste estudo, os autores procuraram verificar as potencialidades do OA desenvolvido para auxiliar os alunos em suas estratégias de resolução de problemas para a construção de conhecimentos sobre conceitos de razão e proporção, com a aplicação de conceitos sobre grandezas diretamente e inversamente proporcionais.

\section{Procedimentos Metodológicos}

A abordagem da pesquisa é de natureza exploratória e do tipo descritiva, foi desenvolvida por meio da utilização de técnicas bibliométricas. Para Vergara (1997), a pesquisa é descritiva quando expõe as características de determinado fenômeno e pode ampliar-se para o estabelecimento de correlações entre fatores ou variáveis, ou ainda, para definição da natureza de tais correlações. Não tem, contudo, o comprometimento de explicar os fenômenos que descreve.

A bibliometria baseia-se na análise estatística da produção científica na forma de artigos, publicações, citações, patentes e outros indicadores mais complexos (OKUBO, 1997). Para Santos e Kobashi (2009) as técnicas bibliométricas são ferramentas embasadas em um arcabouço teórico reconhecido no meio científico que se utiliza de métodos estatísticos e matemáticos para mapear informações, a partir de registros bibliográficos de documentos armazenados em bases de dados.

O desenvolvimento desta pesquisa bibliométrica consistiu em três etapas: coleta de dados, análise de dados e síntese e representação dos dados. Essas três etapas podem ser resumidas nos seguintes passos:

1) Identificação da base de dados: a SCOPUS é uma base de dados multidisciplinar, mantida pela editora Elsevier desde o ano de 2004. Contem mais de 27 milhões de resumos de artigos, referências e índices da literatura científica, técnica e 
médica sendo atualizada diariamente (MESQUITA et al, 2006). Para o campo de estudos de TV Digital, esta base de dados é, possivelmente, a mais representativa por conter os resumos das publicações da IEEE (Institute of Electrical and Electronic Engineers).

2) Definição dos critérios de busca: A estratégia para buscar os artigos científicos em potencial para esta pesquisa teve os seguintes critérios de inclusão:

(a) Artigos que contivessem em seu título, nas suas palavras-chave, nas palavras-chave indexadas pelo SCOPUS ou em seu Abstract termos TV, television, digital, IDTV, DTVI, DTV, learning, education, distance; (b) Foi delimitado o universo temporal de artigos publicados entre os anos de 2000 e 2011 na base de dados SCOPUS que constassem em todas as 4 áreas do conhecimento (Ciências da Vida, Ciências Médicas, Ciências Físicas e Ciências Sociais e Humanas) e suas subáreas; (c) Após, foi realizada uma busca computadorizada utilizando os termos mencionados no item "a" e combinados de acordo com a Figura 1; (d) O resultado dessa estratégia de busca retornou 67 registros, mas dois foram excluídos porque se tratavam de Conference Reviews, ou seja, revisões de conferências e não de artigos científicos. O conjunto inicial de 65 artigos foi então utilizado como base para as análises futuras.

(TITLE-ABS-KEY("TV" OR "television") AND TITLE-ABS-KEY("digital" OR "IDTV" OR "DTVI" OR "DTV") AND TITLE-ABS-KEY("learning" or "education") AND TITLEABS-KEY("DISTANCE")) AND PUBYEAR > 1999 AND PUBYEAR < 2012

Figura 1 - Critérios da busca no SCOPUS

3) Análise dos dados: o conjunto dos dados foi exportado do SCOPUS para utilização com a planilha Excel, de forma a facilitar as análises dos artigos. A análise constou da elaboração de tabelas e figuras para as representações bibliométricas.

Justifica-se a escolha do limite temporal inicial para a realização da pesquisa como o ano 2000, pois a data de implantação de um dos primeiros sistemas foi em 1999 que permitiu o desenvolvimento de serviços e aplicações interativas. O padrão DVB-T (europeu) passou a operar em 1999 no Reino Unido, assim a pesquisa tem o universo temporal de 2000 até 2011. Ressalta-se que os artigos buscados não se limitarão apenas aos que contenham o padrão DVB-T, mas a todos os artigos que tratem de aplicações e serviços de educação a distância via TV Digital, independente do padrão utilizado.

\section{Apresentação e análise dos resultados}

Os dados gerais da pesquisa constam de 65 artigos indexados à base SCOPUS, que foram escritos por 165 autores vinculados a 77 instituições de 24 diferentes países. A quantidade total de referências citadas pelos 65 artigos foi de 877, ou seja, em média os artigos contêm 13 referências. O total de fontes de publicação foi de 56 , sendo que a distribuição dos tipos de publicações mostra o predomínio de artigos publicados em Anais de Eventos (conferências, simpósios ou encontros científicos) com 43 ocorrências, e 20 artigos publicados em periódicos científicos (30,7\%) e 2 artigos classificados como Review. Os dados gerais da análise bibliométrica são apresentados na Tabela 1. 
Tabela 1 - Resumo dos Resultados Gerais do Levantamento Bibliométrico

\begin{tabular}{lc}
\hline Critérios & Frequência \\
\hline \hline Artigos & 65 \\
Autores & 165 \\
Instituições & 77 \\
Países & 24 \\
Referências Citadas & 877 \\
Palavras-chave & 284 \\
Fontes de publicação & 56 \\
\hline \hline
\end{tabular}

A Figura 2 apresenta a distribuição de frequência de publicações ao longo dos anos, com destaque para o maior número de artigos publicados em 2006 (9 artigos), 2008 (10 artigos) e os anos de 2010 e 2011 com 8 artigos cada.

No contexto desta pesquisa, a primeira publicação foi de Leban (2000). O artigo apresenta um assistente de aprendizagem personalizado baseado em Web desenvolvido como um protótipo de um aplicativo de ensino à distância, que utiliza um dispositivo de armazenamento multimídia multiuso para armazenar uma enorme quantidade de dados multimídia localmente no set-top-box, com funções avançadas e também conexão com a Internet. O sistema denominado Personal Learning Assistant (PLA) e o aplicativo permitem interoperabilidade entre a Internet e DVB para que cursos interativos da Web possam ser enriquecidos por vídeos de programas educacionais de TV.

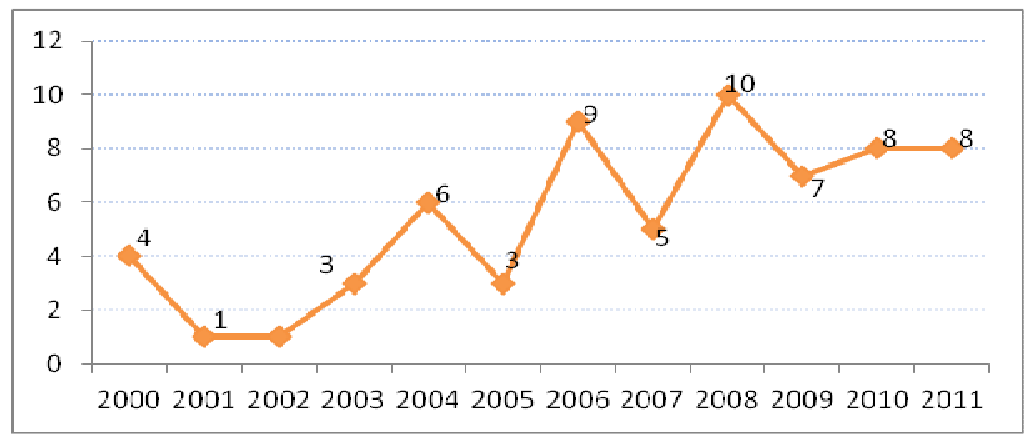

Figura 2 - Publicações por ano

Quando se analisa as publicações por país, o Brasil se destaca porque possui a maior quantidade de artigos do universo desta pesquisa, com 10 publicações (15,3\%). Em seguida estão Espanha e Estados Unidos com 8 artigos cada, e o Reino Unido com 3. Verifica-se que há uma concentração das publicações nos 4 primeiros países que corresponde a 44,6\% dos artigos aqui analisados e uma dispersão nas publicações entre os demais 20 países que publicaram apenas 1 ou 2 artigos cada.

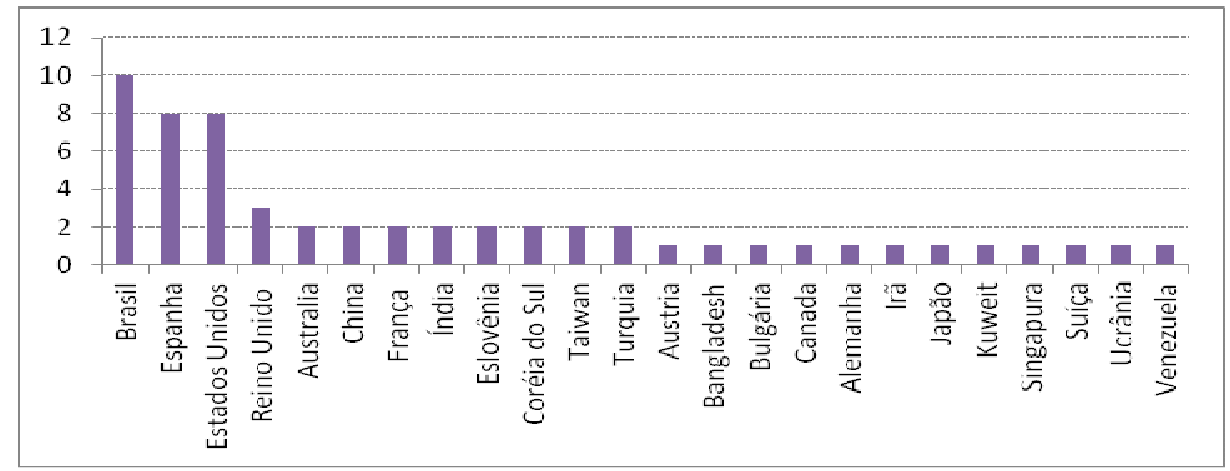

Figura 3 - Distribuição das publicações por país 
A primeira publicação brasileira indexada nesta base da pesquisa foi Tavares et al (2004), que apresenta uma proposta para programas de TV interativos, denominada de "TV imersiva". Foi desenvolvido o aplicativo "Torcida Virtual" para um programa de entretenimento, no qual grupos de espectadores podem participar de atividades coletivas e cooperativas nas plataformas de TVDI, compartilhando o mesmo espaço acústico. Guido Lemos de Souza Filho e Jorge Fernandes são coautores deste artigo e se destacam como pesquisadores muito ativos na área de aplicações em TV Digital, pois são os idealizadores e desenvolvedores do Ginga, ou middleware brasileiro para TV Digital.

O segundo artigo brasileiro desta busca, Sousa et al (2005) desenvolveram um aplicativo para a descoberta de conteúdos educacionais, utilizando o sistema de transmissão que estava ainda em fase de definições na época de publicação do artigo, ou seja, o sistema ISDB japonês, mas acabou por ser adotado pela norma brasileira para a TV Digital com a implantação do SBTVD. Este artigo teve coautores de outros países: França e Canadá e foi financiado pela FINEP e CNPq, como parte dos estudos desenvolvidos por universidades e institutos de pesquisa, que concorreram em um processo licitatório. A chamada pública desse processo foi composta por um conjunto de cartas-convite associadas a requisições formais de proposta (BARBOSA FILHO, 2007).

O artigo de Gomes, Lima e Nevado (2006) apresenta-se como a terceira publicação do Brasil e se assemelha muito à pesquisa realizada por Gomes e Lima (2005), apresentada no item 2.

Santos, Vale e Meloni (2006) apresentam os potenciais que aplicações de $t$ learning podem ter em países com alta exclusão digital, como é o caso do Brasil e também as limitações de um ambiente de aprendizagem pela TV, com base em suas experiências anteriores com programas de ensino aprendizagem baseados na Web. Na criação de aplicações educacionais para TVDi, é recomendado que estas sejam consideradas de forma coletiva, já que os hábitos de assistir à televisão geralmente são realizados como uma atividade de grupo. Assim, tais aplicações devem explorar grupos de trabalho com atividades de colaboração e cooperação entre os estudantes.

Outra limitação apresentada por Santos, Vale e Meloni (2006) é a distância da tela, pois os usuários estão mais distantes dos televisores do que estariam de monitores de computadores. Estratégias de que mantenham a atenção espectador, tais como: fontes de texto claros e grandes, texto espaçado e dividido em pequenas partes, uso de sons, cores e imagens que atraiam a atenção do usuário são algumas das recomendações para desenvolver aplicações de t-learning.

O artigo do Brasil mais recente da base, Silva et al (2011), apresenta uma extensão do SCORM - um padrão de referência para interoperabilidade dos objetos de aprendizagem de e-learning - com o objetivo de adaptá-lo para melhorar a pesquisa e navegação para OAs de $t$-learning. Eles apresentam a ferramenta de autoria denominada "Adaptador T-SCORM" para aplicar esta extensão de um modo rápido e eficiente.

Dos 10 artigos publicados por instituições brasileiras mencionados na figura 3 , somente a Universidade de São Paulo possui 5 publicações (50\%) e também aparece como a segunda colocada dentre as 10 instituições com maior quantidade de artigos publicados nesta pesquisa (conforme tabela 2). 
Tabela 2 - As 10 instituições com maior quantidade de artigos sobre "t-learning"

\begin{tabular}{clc}
\hline \hline Nr. & Instituição & Qtde. Publicações \\
\hline \hline 1 & Universidad de Vigo (Espanha) & 6 \\
2 & Universidade de São Paulo (USP) & 5 \\
3 & Hewlett Packard Laboratories (Estados Unidos) & 2 \\
4 & Tamkang University (Taiwan) & 2 \\
5 & Universidade Federal do Rio Grande do Norte (UFRN) & 2 \\
6 & University of Southern California (Estados Unidos) & 2 \\
7 & University of Ljubljana (Slovênia) & 2 \\
8 & Instituto Federal de Educação de Manaus (AM) & 1 \\
9 & Centro Federal de Educação Tecnológica do Ceará & 1 \\
10 & Gangwon National University (Coréia do Sul) & 1 \\
\hline \hline
\end{tabular}

A Universidade do Vigo é a primeira colocada entre as instituições que mais publicaram sobre t-learning no SCOPUS, com 6 artigos e destacam-se 4 instituições brasileiras (USP, UFRN, IFET-AM e CEFET-CE) no conjunto das 10 instituições que mais publicaram. Tal fato demonstra que as políticas públicas para incentivo às pesquisas em TV digital interativa com foco na inclusão social e na educação a distância (BARBOSA FILHO, 2007; ZUFFO, 2008) tem se desdobrado em pesquisas de destaque no cenário internacional.

Nota-se que há uma dispersão das pesquisas por diferentes universidades e algumas empresas ou centros de pesquisas vinculados a empresas, como a $H P$ Laboratoires, que possui 2 publicações e outras empresas como Nokia, Alcatel, Motorola, IBM Research Center que possuem 1 publicação cada.

$\mathrm{Na}$ Espanha, todas as publicações foram realizadas pelo departamento de Engenharia Telemática da Universidade de Vigo, cujos autores também são referências em pesquisas sobre t-learning. Pazos-Arias et al (2008) possui 14 citações na base SCOPUS e aparece como segundo mais citado dentre os 65 artigos desta base.

Os autores delimitaram o âmbito do t-learning, em comparação com a aprendizagem baseada na web e analisaram a influência da normalização da TVDi como plataforma de serviços educacionais, introduziram um quadro para o desenvolvimento e implantação de serviços de $t$-learning que promove a interoperabilidade e a reutilização dos OAs tendo em conta as características do da TVDi para o cenário europeu, ou seja, para o padrão DVB (PAZOS-ARIAS et al, 2008).

Outros 2 artigos da Universidade do Vigo estão entre os 10 artigos mais citados no SCOPUS desta base de 65: Pazos-Arias et al (2006) que teve 5 citações e LópezNores et al (2003) com 4 citações.

Pazos-Arias et al (2006) apresentam o ATLAS, um framework para o desenvolvimento e implantação de serviços de t-learning para multiusuários. Eles abordam a arquitetura para os serviços e procuram demonstrar a ferramenta que permite que os serviços possam ser criados com um conhecimento mínimo de programação.

López-Nores et al (2003) exploraram o desenvolvimento de aplicativos de $t$ learning com base no padrão MHP, com a apresentação de uma ferramenta baseada em XML, eles também defendem que a ferramenta facilita desenvolver aplicações sem a necessidade de conhecimentos prévios de programação, similar ao ATLAS.

Outro dado relevante apresentado pela base SCOPUS, diz respeito às subáreas de conhecimento dos artigos publicados, sendo que um artigo poderá pertencer a mais de uma subárea, conforme o gráfico 3. 


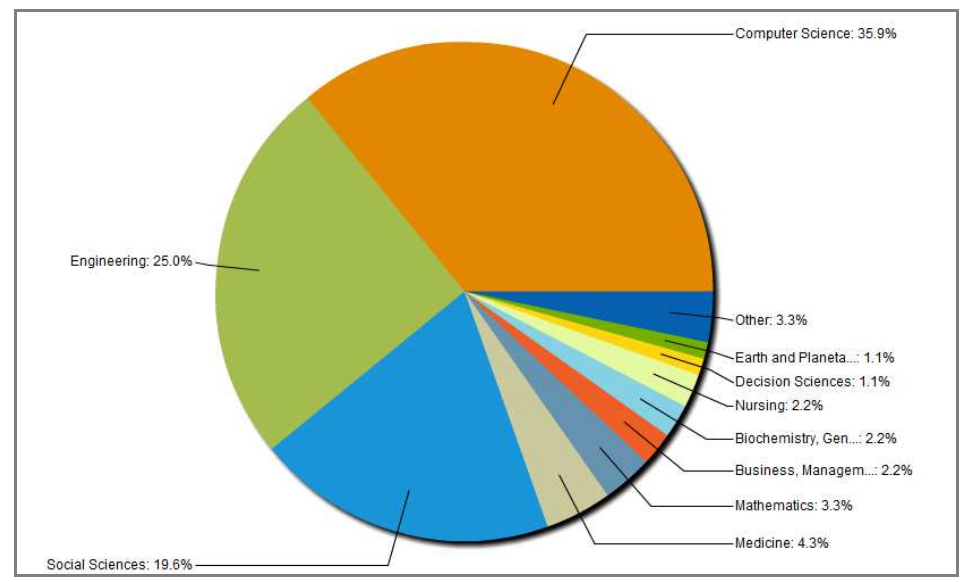

Figura 4 - Subáreas do conhecimento das publicações em t-learning no SCOPUS

Nota-se que as principais áreas que estão pesquisando o assunto de t-learning são Ciências da Computação com 35,9\% dos artigos; Engenharia que teve 25\% das publicações e Ciências Sociais com 19,6\%. Com base nos dados verifica-se que o tema tem sido mais abordado pelas áreas tecnológicas do que as sociais ou humanísticas. Como as publicações podem estar associadas em mais de uma área de conhecimento, indicam a natureza multidisciplinar do tema.

Observou-se boa parte dos trabalhos relacionados às ciências da computação e engenharia tem relação com o desenvolvimento de aplicações para t-learning, ou trata de questões da infraestrutura de recepção e transmissão em TVDI, também fazem parte arquiteturas e protótipos de hardware e softwares para a implementação de aplicações e serviços em contextos educacionais para TV digital.

Muitos dos artigos mostram resultados de testes de sistemas e aplicativos, ou experimentação por parte de usuários, mas em escala pequena, nos laboratórios ou centros de pesquisa, denotando que o t-learning encontra-se no estágio inicial desenvolvimento com as inovações sendo testadas por pesquisadores em grande parte da academia e poucas empresas.

O idioma dos artigos publicados nesta base foi de 61 artigos escritos em Inglês, 2 em Português, 1 em Espanhol e 1 em Polonês. Ainda que pesquisadores de outros países estejam contribuindo para o avanço dos estudos em t-learning, o percentual de 93,84\% de artigos em Inglês deve-se ao fato que boa parte das fontes de publicação (anais de conferências ou periódicos internacionais indexados ao SCOPUS) tenha como língua preferencial o inglês, muitas vezes até como a única língua aceita.

\section{Conclusão e trabalhos futuros}

Esta pesquisa pretendeu avançar no campo de estudos de TV Digital, no que diz respeito ao tema específico de aplicações e serviços em t-learning de forma a proporcionar um ponto de partida para identificação de tendências, sobreposições, lacunas ou desafios para as pesquisas sobre o tema. Assim, foi realizada uma análise bibliométrica dos artigos de t-learning no SCOPUS entre 2000-2011. A combinação dos dados bibliométricos com a análise do conteúdo dos abstracts dos artigos da base selecionada fornece informações valiosas para os educadores e pesquisadores em $t$ learning.

A investigação na literatura científica demonstrou que o t-learning é um campo de pesquisa em amplo crescimento nos últimos anos. A participação de pesquisadores de instituições brasileiras no campo demonstra a aplicação dos recursos financeiros 
provenientes das políticas públicas de implantação do SBTVD. Os países com maior destaque desta pesquisa foram Brasil e Espanha, ainda que haja uma dispersão entre as publicações em nível mundial e as áreas predominantes de pesquisa são as tecnológicas, mesmo que o tema tenha se desenvolvido por uma abordagem interdisciplinar.

Contudo, não se deve apenas focar nos aspectos tecnológicos, mas também nas novas práticas educacionais que os recursos tecnológicos disponibilizam. A evolução histórica das publicações analisadas demonstra que o desenvolvimento da pesquisa sobre t-learning tende a estar mais focada nas tecnologias (desenvolvimento de aplicações, usabilidade, ergonomia etc.) do que nas práticas pedagógicas.

Como sugestão para trabalhos futuros, cabe uma investigação sobre os usos educacionais do t-learning, sobre as metodologias de ensino e efeitos destes novos recursos no processo de ensino e aprendizagem, pois além dos aspectos tecnológicos e educacionais, a pesquisa poderia se ampliar para os efeitos que as tais práticas educacionais possam ter sobre os indivíduos nas suas relações sociais.

\section{Agradecimentos}

Esta pesquisa foi financiada pela Coordenação de Aperfeiçoamento de Pessoal de Nível Superior (CAPES), através do Programa de Formação de Recursos Humanos em Televisão Digital (RH-TVD/CAPES).

\section{Referências Bibliográficas}

AARRENIEMI-JOKIPELTO P. T-learning Model for Learning via Digital TV. 16th EAEEIE Annual Conference on Innovation in Education for Electrical and Information Engineering (EIE), Lappeenranta, Finland, 2005.

BARBOSA FILHO, André. O Sistema Brasileiro de Televisão Digital: do sonho à realidade. In: TV digital: qualidade e interatividade. Brasília : IEL/NC, 2007.

BATES, Peter J. T-learning Study: A study into TV-based interactive learning to the home. PJB ASSOCIATES (Inglaterra), 2003.

BELLOTTI et al. A T-learning Courses Development and Presentation Framework. IMCL International Conference on Mobile and Computer aided Learning. 2008, April 16-18, Amman, Jordan.

BOLAÑO, C. ; VIEIRA, V. G. R. . TV digital no Brasil e no mundo: estado da arte. Eptic On-Line (UFS), Aracajú, SE, v. VI, n. 2, p. 102-134, 2004.

CROCOMO, F. TV Digital e produção interativa: a comunidade manda notícias. Florianópolis: UFSC, 2007.

DIAS, F. R.; RODRIGUES, A.; FARIAS JUNIOR, I. H. Estudo de Caso: EAD Através da TV Digital Interativa com o Software Trânsito Legal. RENOTE - Revista Novas Tecnologias na Educação. Porto Alegre. v. 8, n. 1, 2010.

FECHINE, Y.; FIGUERÔA, A. Cinema e Televisão no Contexto da Transmediação. In: RIBEIRO, A. P. G.; SACRAMENTO, I.; ROXO, M. (org.). História da Televisão no Brasil. São Paulo: Contexto, 2010. p. 281-311.

GOMES, F. J. L; LIMA, J. V.. O papel como interface para o t-learning. RENOTE Revista Novas Tecnologias na Educação. Porto Alegre. v. 3, n. 2, nov. 2005.

GOMES, F. J. L, LIMA, J.V.; NEVADO, R.A. O papel comum como interface para TV digital, Proceedings of the VII Symposium on Human Factors in Computing Systems, IHC 2006, pp. 29. 
GOMES, F. J. L; LIMA, J. V.; NEVADO, R. A. Avaliando um Objeto de Aprendizagem para TV Digital. RENOTE - Revista Novas Tecnologias na Educação, 2009

LEBAN, M. DVB/Internet interoperability and new possibilities in distance learning, International Symposium on Technology and Society, 2000, pp. 207.

LÓPEZ-NORES, M., et al. A mixed XML-JavaBeans approach to developing t-learning applications for the multimedia home platform. Lecture Notes in Computer Science, pp. 376-387, 2003.

LYTRAS, M. et al. Interactive Television and E-learning Convergence: Examining the Potential of T-learning. ECEL2002, The European Conference on e-learning, Brunel University, UK, 2002.

MELO, B. R. S. et al. Objeto de Aprendizagem Gangorra Interativa na Compreensão Conceitual de Grandezas. Anais do XXVIII do Congresso da SBC (CSBC 2008), WIE - Workshop sobre Informática na Escola. Belém, PA, 2008.

MESQUITA, Rosa et al. Elaboração e aplicação de instrumentos para avaliação da base de dados Scopus. Perspect. Ciênc. Inf., Belo Horizonte, p. 187-205. Maio-Agosto 2006.

OKUBO, Y. Indicateurs bibliométriques et analyse des systèmes de recherche: méthodes et exemples. Documents de travail sur la science, la technologie et l'industrie. Paris: OCDE, 1997.

PAZOS-ARIAS, J.J., et al. Provision of distance learning services over Interactive Digital TV with MHP. Computers and Education, vol. 50, no. 3, 2008, pp. 927-949.

PAZOS-ARIAS, J.J., et al: A framework to provide multiuser and distributed t-learning services over MHP, Software - Practice and Experience, vol. 36, no. 8, 2006, pp. 845869.Porto Alegre. v. 7, n. 3, dez. 2009.

ROESLER, V. et al. Um novo modelo educacional através da TV Digital. RENOTE Revista Novas Tecnologias na Educação. Porto Alegre. v. 8, n. 2, jul 2010.

SANTOS, D.T., VALE, D.T. \& MELONI, L.G.P. Digital TV and distance learning: Potentials and limitations, Proceedings - Frontiers in Education Conference, FIE. 2006.

SANTOS, R. N. M.; KOBASHI, N. Y. Bibliometria, cientometria, infometria: conceitos e aplicações. Pesq. bras. Ci. Inf., Brasília, v. 2, n. 1, p. 155-172, 2009.

SILVA, F.M. et al. Extending the SCORM standard to support the project of educational contents for t-Learning. Advances in Intelligent and Soft Computing, 2011, pp. 293-302.

SOUSA, V.P. et al. An API for the discovery of educational content on the Brazilian digital TV, ITHET 2005: 6th International Conference on Information Technology Based Higher Education and Training, 2005, pp. T4B.

TAVARES, T.A. et al. Sharing virtual acoustic spaces over interactive TV programs Presenting "virtual cheering" application, IEEE International Conference on Multimedia and Expo (ICME), 2004, pp. 2175.

VERGARA, S. C. Tipos de pesquisa em administração. Cadernos EBAP, FGV, Rio de Janeiro, n. 52, jun 1997.

ZUFFO, M. K. TV Digital Aberta no Brasil - Políticas Estruturais para um Modelo Nacional. Disponível em: Acesso em: 20 de outubro de 2008. 